\title{
LIV. Note on the conditions of self-excitation in a dynamo machine
}

\section{Silvanus P. Thompson D.Sc.}

To cite this article: Silvanus P. Thompson D.Sc. (1888) LIV. Note on the conditions of selfexcitation in a dynamo machine, Philosophical Magazine Series 5, 26:163, 469-475, DOI: 10.1080/14786448808628301

To link to this article: http://dx.doi.org/10.1080/14786448808628301

曲 Published online: 29 Apr 2009.

Submit your article to this journal $\sqsubset \pi$

Џ Article views: 2

Q View related articles $\asymp$ 


\section{LONDON, EDINBURGH, AND DUBLIN \\ PHILOSOPHICAL MAGAZINE}

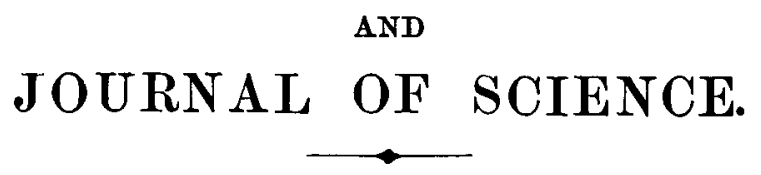

[FIFTH SERIES.]

$D E C E M B E R \quad 1888$.

LIV. Note on the Conditions of Self-Excitation in a Dynamo Machine. By Silvanus P. Thompson, D.Se.*

W HEN about the year 1867 it began to be recognized that a machine constructed on the principle of magnetoelectric induction might be made to excite the magnetism in its own field-magnets, it became usual to explain the phenomenon of self-excitation in language something like the following. There being a small amount of residual magnetism in the core of the stationary electromagnet, very feeble currents will be induced in the secondary or armature-coils when they are set into rotation at any given speed. If these feeble induced currents are now sent round the coil of the fixed electromagnet they will exalt its feeble magnetism, and its inductive action will be stronger ; these stronger currents being also sent round the magnet will raise its magnetism still higher, and so by action and reaction the magnetism and the currents grow at a compound-interest rate until the limit fixed by the magnetic saturation of the iron core of the field-magnet is reached.

Long before the true rationale of the limit of self-excitation was assignable, it was known that this method of considering the subject was faulty. Were it true, it would follow that, no matter what the speed of driving, or the resistance in the electric circuit, the magnetism would go on rising until the iron had attained saturation. The facts, on the contrary, are (1) that in self-exciting machines the degree of excitation of the field-magnets depends most emphatically on the speed and

* Communicated by the Physical Society: read May 12, 1888.

Phil. Mag. S. 5. Vol. 26. No. 163. Dec. $1888.2 \mathrm{~K}$ 
on the resistances of the electric circuit; (2) that for machines which have their exciting coil in series with the armature and main circuit, there is a certain relation between speed and resistance such that they do not excite themselves at all unless the quotient of speed by resistance attains a certain minimum value ; (3) that in machines which have their exciting coil connected as a shunt to the main circuit there is again a certain relation between the speed and the electric resistances of the various parts of the circuit, such that with a given speed self-excitation does not take place unless the resistance of the external circuit exceeds a certain limiting value.

On June 26, 1886, I had the honour of bringing before the Physical Society* some formula relating to the performance of dynamo-electric machines, in which this matter was touched upon, and in which, on the assumption that the law of magnetization might be adequately represented by the LamontFrölich formula, expressions were obtained for the relations between speed, magnetization, resistance, and current. As an important conclusion it was shown, always upon the above assumption, that at a given speed of driving and with a given resistance in the electric circuit, the current, of any given series-wound machine, was always less by a certain fixed quantity than that current which would have been produced (at that speed and with the given resistance) had the magnetism been actually at full saturation. This difference between the actual current and the current that there would have been at saturation was a definite number of amperes, and was shown to be that number of amperes which would have been sufficient to produce exactly half-saturation in the given machine. This half-saturating number of amperes I had previously denominated the "Diacritical" current. This proposition having been established, it became obvious that the machine would not excite itself if the speed and resistance were such-the speed so low or the resistance so high-that the current which would have been produced with fieldmagnet independently saturated was less than the diacritical current. Or, if the resistance of the circuit were prescribed, there would obviously be a least-speed of self-excitation.

The assumption in the above investigation of an approximate expression for the law of magnetization necessarily made the argument less satisfactory than would be the case were no such assumption made. In the argument which follows no assumptions are made of an empirical character as to the law of magnetization, absence of secondary phenomena,

* See Phil. Mag. September 1886. 
such as electric and magnetic leakage, being the only point assumed.

It is known that the electromotive force of a dynamo may be written

$$
\mathrm{E}=n \mathrm{CN} ; . . . \quad . \quad . \quad .
$$

where $\mathrm{E}$ is the electromotive force (in absolute C.G.S. units); $n$ the number of revolutions of the armature per second; $\mathrm{C}$ the number of conductors counted round the periphery of the armature, and closed in series; $\mathrm{N}$ the whole number of magnetic lines (in C.G.S. units) that traverse the armature.

So, writing $\Sigma R$ for the sum of the electric resistances (in C.G.S. units) of the circuit in which the electromotive force operates, the current flowing in the circuit may be written as

$$
i=\frac{n \mathrm{CN}}{\Sigma \mathrm{R}} \text {. . . . . . . . }
$$

Again, it is known that the number of magnetic lines in the armature may be calculated by dividing the line-integral of the magnetizing forces by the sum of the magnetic resistances of the " magnetic circuit" of the dynamo. If the machine is joined up with its exciting coil of $\mathrm{S}$ convolutions in series with the main eircuit, the line-integral of the magnetizing forces will be written as $4 \pi \mathrm{S} i$. Then, writing $\Sigma \rho$ as the sum of all the magnetic resistances in the magnetic circuit, we shall have

From (2) we get

$$
\mathrm{N}=\frac{4 \pi \mathrm{S} i}{\Sigma \rho} \cdot \cdot \cdot \cdot \cdot \cdot \cdot \cdot
$$

$$
\frac{i}{\mathrm{~N}}=\frac{n \mathrm{C}}{\mathrm{\Sigma L}} ; \cdot \cdot \cdot \cdot \cdot \cdot \cdot \cdot .
$$

and, from (3),

$$
\frac{i}{\mathrm{~N}}=\frac{\Sigma \rho}{4 \pi \mathrm{S}^{*}} \cdot \cdot \cdot \cdot \cdot \cdot \cdot \cdot \cdot
$$

Equating (4) and (5), we have

$$
4 \pi \mathrm{S} n \mathrm{C}=\Sigma \rho . \Sigma \mathrm{R} . \quad . \quad . \quad . \quad . \quad
$$

It will be noticed that this equation contains neither $\mathrm{N}$ nor $i$, and its truth is independent of the relation between $i$ and $\mathrm{N}$. It is true that $\Sigma \rho$, which is a factor of the expression on the right-hand side, depends on $\mathrm{N}$, and, in general, increases when $\mathrm{N}$ is increased. But the truth of the equation is not affected by the form of the relation between $\Sigma \rho$ and $N$; in brief, it is true independently of any assumptions as to the form of the law of magnetization.

On examining the expression on the left hand of the equa$2 \mathrm{~K} 2$ 
tion, it will be seen that, for a given dynamo, all its factors, except the speed $n$, are invariable. Hence the main fact brought out by this equation is that in a series dynamo, driven at constant speed, the product of the magnetic resistance into the electric resistance is a constant. We have here the key to the behaviour of the self-exciting dynamo ; - the explanation why it is that the machine, though exciting itself up "at a compound-interest rate," stops short of absolute saturation in the degree to which its magnetization is raised. As the field-magnets get more and more highly magnetized, the magnetic resistance increases. Suppose that such a dynamo, running at a constant speed, works at a certain degree of magnetization when there is a certain resistance in the electric circuit. Suppose that resistance to be now reduced: the current increases; as a result there is higher magnetization, higher electromotive force, and therefore still greater current. To what point will these things go on increasing? Equation (6) gives the answer: until the decrease in the electric resistance is exactly balanced by the increase in the magnetic resistance.

Other phenomena of the action of the dynamo now become more intelligible. As is well known, in ascending magnetizations of iron there is a certain stage at which the magnetic permeability increases, instead of decreasing, with an increase in the magnetization. In a dynamo at this stage of excitation, any increase in the electric resistance, causing a decrease in the current, will also cause an increase in the magnetic resistance; hence this stage of excitation is one of instability. The dynamo is either excited above this stage, or else its excitation falls below this stage-virtually to zero.

The two factors of the product $\Sigma \rho . \Sigma R$ are both complex quantities, containing constant as well as variable terms. We may write

$$
\Sigma \rho=\alpha+\xi \text {. }
$$

Here the constant part $\alpha$ relates to the magnetic resistance of the gap between the armature-core and the polar surface of the field-magnets; which gap, filled partly with copper, partly with air, and partly with insulating materials, possesses, so far as is known, a constant magnetic resistance. The variable part $\xi$ of the magnetic resistance relates to the iron portions of the magnetic circuit: it is in general the sum of a number of terms such as $l / \mu \mathrm{A}$, where $l$ is the length, A the sectional area, and $\mu$ the permeability, for the time being, of the various portions-cores, yoke, \&c.

Similarly, we may write

$$
\Sigma \mathrm{R}=a+x
$$


where $a$ stands for the constant part of the electric resistances (armature and main-circuit coils) in the circuit, and $x$ for the variable part; that is to say, the resistance of the external circuit, including under that term not only true ohmic resistances, but also the effects of counter electromotive forces opposed in the path of the current of the machine.

We may now rewrite equation (6) in the following form for the series-wound machine driven at a constant speed :-

$$
(\alpha+\xi)(a+x)=\text { constant. . . . . }
$$

Now at starting, when as yet the magnetization of the iron is almost zero, the resistance of the iron part of the magnetic circuit is small. Its value is not indeed quite zero, but depends upon the prior history of the iron. It is certainly so small as to be negligible compared with the value of $\alpha$. Hence there will be a particular maximum value of $x$, which we may call $x_{1}$, corresponding to the case when $\xi=0$. This maximum value, which will be given by the equation

$$
a_{1}=\frac{4 \pi n \mathrm{CS}}{\alpha}-a, \text {. . . . . . }
$$

will be the critical external resistance of the machine at the prescribed speed. If any greater resistance than $x_{1}$ be intercalated in the circuit the machine will refuse to excite itself, even though there is a small initial magnetization present. So far as there is a weak magnetization present, the machine will act as a feeble magneto-electric machine.

Suppose, now, that $x$ is so far decreased that the total electric resistance is reduced to half its limiting value, that is to say, let $x^{\prime}=\frac{1}{2} x_{1}-\frac{1}{2} a:$ then the current and the magnetization will both run up until the magnetic resistance is equal to double its initial value, and $\xi$ will have the value $\xi^{\prime}=\alpha$. The permeability of the magnetic circuit, taken as a whole (iron, copper, and air), will have been halved. This state of things corresponds to the "diacritical point" in the excitation first investigated by the author in 1884; and, if the LamontFrölich formula were true, this point would correspond to the state of semi-saturation of the magnetization.

Again, suppose the variable part of the electric resistance to be reduced to zero; in other words, let the machine be short-circuited. Then $\xi$ will run up to the maximum value it can have at that speed. Calling this value $\xi_{2}$, we have

$$
\xi_{2}=\frac{4 \pi n \mathrm{CS}}{a}-\alpha . \quad \text {. . . . . }
$$

It is further clear that if a fixed value be given to the 


\section{Conditions of Self-Excitation in a Dynamo Machine.}

external electric resistance $x$, there will be a limit in speed below which the machine will refuse to excite itself, the leastspeed of self-excitation (called by some authorities the "dead turns," by others the "critical velocity") being given by the equation

$$
n_{1}=\frac{\alpha(a+x)}{4 \pi \mathrm{CS}} \cdot \text {. . . . . }
$$

This throws some light on the observed fact that very small machines are not self-exciting except at very high speeds. The fixed part $\alpha$ of the magnetic resistance varies in similar machines of different size, inversely as the linear dimensions; also the fixed part $a$ of the electric resistance varies inversely as the linear dimensions of the machine. Hence the leastspeed of self-excitation in similar machines (on short-circuit) varies inversely as the square of the linear dimensions. In practice it is possible, in larger sizes of machine, to reduce both $\alpha$ and $a$ to amounts relatively smaller; for the actual air-space left for clearance may be relatively smaller in large machines, and a relatively greater thickness of copper on the armature-periphery is admissible. When the machine is on short-circuit, $x=0$, and the last equation then becomes

$$
n_{1}=\frac{\alpha a}{4 \pi \mathrm{CS}} \cdot \text {. . . . . . }
$$

This, too, is important as showing that the least-speed of selfexcitation of a machine while short-circuited is a measure of the goodness of the magnetic circuit.

For a shunt-wound dynamo the fundamental equation becomes

$$
(\alpha+\xi)\left(a+s+\frac{a s}{x}\right)=4 \pi n \mathrm{CZ} ; \quad . \quad .
$$

where $a$ is, as before, the resistance of the armature-circuit within the machine, $s$ the resistance of the exciting shuntcircuit, and $\mathrm{Z}$ the number of convolutions of the shunt-coil. Excitation does not take place at a given speed $n$ unless $x$ has as its minimum value

$$
x=\frac{\alpha a s}{4 \pi n \mathrm{CZ}-\alpha(a+s)}, . . \quad .
$$

On open circuit, when $x=\infty$, the least-speed of self-excitation is

$$
n_{1}=\frac{\alpha(a+s)}{4 \pi \mathrm{CZ}}, . \quad . \quad . \quad . \quad .
$$

the machine then exciting itself through its own circuit as a 
series-machine. If short-circuited it will require an infinite speed to become self-exciting.

There is a still more general way of establishing the relation (6). Every dynamo may be regarded as an arrangement of two circuits, a primary (or field-magnet) circuit and a secondary (or induced or armature) circuit, between which there is a coefficient of mutual induction $M$, which is caused to vary by mechanically altering the configuration of the system. If the currents in the two circuits be called respectively $i_{1}$ and $i_{2}$, then the work done in starting or stopping either current in the presence of the other is equal to $\mathbf{M} i_{1} i_{2}$. Now in each revolution of the armature of an ordinary dynamo the current in each of the sections of the armature-coil is twice started and twice stopped. Hence in each semirevolution the work to be mechanically performed in driving is equal to $\mathrm{M} i_{1} i_{2}$; or if, as in the series-dynamo, the current in the two circuits is the same, the work to be done is $\mathbf{M} i^{2}$. Now, on the assumption that there is no magnetic leakage, the value of $M$ is equal to $4 \pi S_{1} S_{2} \div \Sigma \rho$; where $S_{1}$ and $S_{2}$ are the respective numbers of convolutions of the primary and secondary coils which surround the tubes of magnetic force in the magnetic circuit. For our purpose, $\mathrm{S}_{2}$, in ordinary armatures, with symmetrically-wound coils united in a selfclosed circuit, tapped at two opposite points by the two contact-brushes, and with commutation occurring at points where each commuted convolution encloses a maximum magnetic flux, is equal to $\frac{1}{2} \mathrm{C}$. But the work done by the machine in the electric circuit during a semi-revolution is equal to $i^{2} \Sigma \mathrm{R} \div 2 n$. Equating this to the work done on the machine gives, as before,

$$
\Sigma p . \Sigma \mathrm{R}=4 \pi n \mathrm{CS} \text {. }
$$

City and Guilds Technical College,

Finsbury, June 1888.

LV. Remarks on the Weathering of Rocks, and certain Electrical Phenomena, suggested by some Statements in a popular Novel. By Charles Tomlinson, F.R.S.\&c.*

THE demand for works of fiction has been increasing of late years at so considerable a rate that the chief business of a large circulating library consists in dealing out novels to its subscribers. A cursory examination of the contents of a railway bookstall shows by the gaudy covers of its books that cheap reprints of popular novels form the staple commodity.

* Communicated by the Author. 